\title{
Enhancement of Spectrum Efficacy using Cognitive Radio Networks
}

\author{
Vivek Kukreja, Shailender Gupta, Bharat Bhushan, Poonam Mittal and C. K. \\ Nagpal \\ YMCA University of Science and Technology, Faridabad, India \\ dit.vivek@gmail.com,shailender81@gmail.com,bhrts@gmail.com, \\ poonamgarg1984@gmail.com,nagpalckumar@rediffmail.com
}

\begin{abstract}
Spectrum is the most valuable and scarce resource for every wireless application. Due to the scarcity of the spectrum other alternatives are also being searched. Cognitive Radio $(C R)$ technology is one such alternative which has gained popularity in the recent times. It has been established from various researches that the allocated band is not in use for all the time by the licensed user or as Primary Users (PUs). The Cognitive Radio users also called as Secondary Users (SUs) communicate using unlicensed band or by using the idle licensed band of primary users opportunistically. This paper evaluates the performance of routing protocols by varying concentration level of $P U$ and $S U$. We have also investigated the impact of time given by PU's to SU's for data transfer. For performing the above task, a simulator is designed in MATLAB-7.01. The results show that the performance of routing protocols improves significantly as the number of PUs and allowable time given by PU's to SU's increases.
\end{abstract}

Keywords: Cognitive Radio, Routing, Simulation

\section{Introduction}

The explosive growth in high speed internet is spurring a new technology wave. The key ingredient in any wireless applications is the spectrum i.e. the frequencies and airwaves over which the wireless signals get transmitted over. Simply stated the providers can't offer new services without spectrum and enough of it. Spectrum has been under tremendous demand ever since. The reason for the same can be:

- Advancement in technology: Advancement in technology has resulted in design of miniature size, low power, highly integrated and portable wireless devices. It has led to increase in demand for spectrum i.e. smart phone needs 24 times more bandwidth for broadband application.

- Growth in population: The growth in human population created heavy demand for wireless applications which in turn has led to increase in demand of liberal spectrum.

- Growth in wireless application: Considering the advantages of wireless technology, it has witnessed a tremendous growth. Many wired counterparts are also shifting fast to wireless platform. Also $2 \mathrm{G}$ technologies has upgraded to $3 \mathrm{G}$ and $4 \mathrm{G}$ which is spectrum hungry.

Due to above reasons the spectrum demand is increasing at an exponential rate. Many measures are in force to judiciously allocate the spectrum as under:

- Stringent spectrum regulations: The nations have appointed regulators to control allocation of spectrum. Regulators assign spectrum on Fixed Spectrum Assignment (FSA) basis and force stringent measures to curb the demand. This has partially helped in justified use of spectrum. 
- Migration from analog to digital technology: In some nations the analog technology is being replaced with digital technology which is more bandwidth efficient.

- Frequency reuse: It is common technique being utilized in cellular mobile communication. The frequency reuse technique deals with spectrum crunch effectively in many wireless applications.

- In spite of all the above measures to maintain equilibrium between demand and supply, demand for new spectrum is surpassing all effort.

This paper is an effort to analyze the variation in the performance of ad hoc network by introducing the concepts of cognitive radio. Ad hoc network is an infrastructure less network wherein there is no fixed access point or base station. The nodes in ad hoc environment are mobile in nature as a result the topology between them is never fixed. Source to destination communication in such an environment can be achieved through the intermediate nodes available between the two. The cognition property of CR can be used to enhance the spectrum management and spectrum utilization in the ad hoc network.

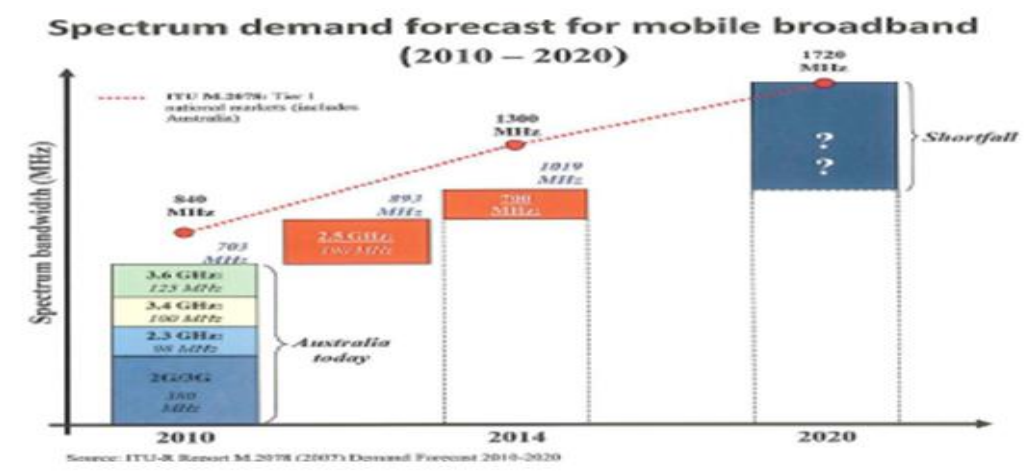

Figure 1. Demand and Supply of Spectrum Availability

In cognitive radio networks (CRN) there are two types of users, Primary Users and Secondary users. The PU [3] is allocated license to use a particular band of frequency while the SU communicate through the licensed spectrum of PU nodes whenever the channel is not occupied by the PU node i.e. the spectrum is used opportunistically by SUs. Whenever the PUs wishes to use the band the SUs release it to avoid collisions or interferences with the PUs. With increase in concentration of PUs and the time allocated by PUs to SUs, the performance of routing protocol should enhance. This paper is an effort to find the impact of the same. The rest of paper has been organized into following sections: Section 2 provides problem definition. Section 3 provides the simulation setup parameters and metrics used to evaluate performance of the network. Section 4 provides results and discussion. Concluding remarks are provided in Section 5 followed by references.

\section{Problem Definition}

Figure 1 shows the demand and supply curve for the year 2010 - 2020. The curve shows that even today we are short of spectrum availability. The situation would worse by the end of this decade. It can be seen that by the year 2020 we will be short of spectrum of nearly $540 \mathrm{MHz}$ as given by ITU-R.

To cater to this huge demand CR networks may be used. In the subsequent section we will see how this technology of CR networks is beneficial for conserving the spectrum. 


\section{Simulation Set Up}

\subsection{Performance Metrics Used}

To evaluate the performance comparison of routing protocol the following performance metrics were used as follows:

- $\quad$ Packet Delivery Ratio (PDR) - Defined as the ratio of total packets received by the source to the total number of packets transmitted.

- Hop Count- Defined as the number of intermediate hops from Source to destination.

- $\quad$ Probability of Reach ability (PoR) - Defined [15] as the ratio of possible reachable routes to the all the possible routes between all possible source and destinations pairs.

- Path optimality- Defined as the ratio of total distance travelled in cognitive network to the distance travelled in ad hoc network (The network in which PU simply drops the control/data packets of SU).

\subsection{Simulator Design}

A Simulator was been designed in MATLAB to study the impact of varying relative concentration of PUs and SUs and by varying time allocated by PUs to SUs. A region of $2000 * 2000 \mathrm{Sq}$ Units is chosen where 40 Nodes are placed at random positions using Randint () function as shown in the Figure 2. At any instant the path between any randomly chosen source and destination pair is selected by the use of Dijkstra's shortest path algorithm.

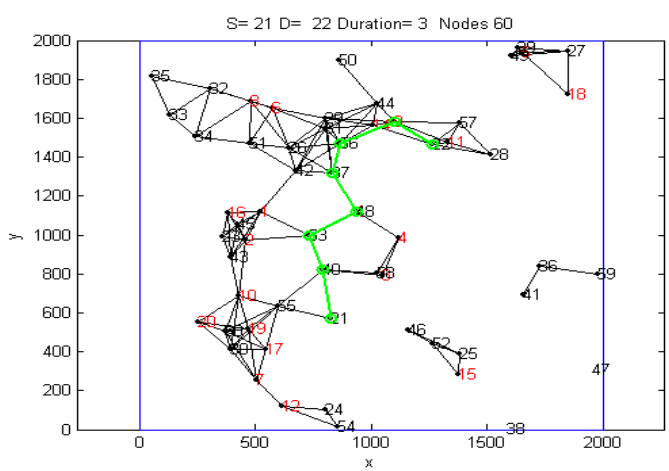

Figure 2 (a). Path with No Intermediate Node as Primary

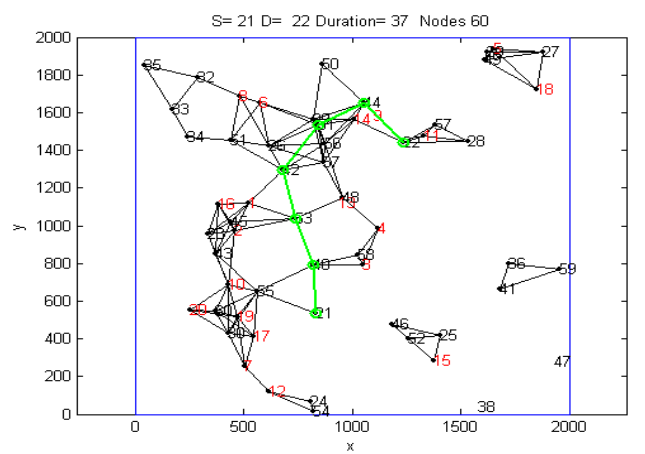

Figure 2 (b). Alternate Path between Same Source and Destination Pair through a PU node 
For a route decided between source and destination pair belonging to SU category, communication will be feasible if path chosen consists of Secondary nodes entirely or combination of PU and SU as intermediate nodes. Communication on a path involving PU as one of the intermediate nodes ceases instantly as the PU gets activated. The alternate path is searched thereafter. PU nodes are shown in red color While SU nodes are shown with black color. It could be clearly seen in Figure $2 \mathrm{a}$ that route is established using SUs only and no PUs are involved as intermediate nodes.

On the other hand Figure $2 \mathrm{~b}$ shows the alternate path between same source and destination pair involving PU nodes as intermediate nodes. This path will persist till the PU nodes are inactive and is discarded as soon as the PU gets activated.

\subsection{Set up Parameters}

Set up parameters used in the simulation are shown in Table 1.

Table 1. Set up Parameters

\begin{tabular}{|l|l|l|l|}
\hline \multicolumn{1}{|c|}{ Set up parameters } & \multicolumn{1}{c|}{ Values } & \multicolumn{1}{c|}{ Set up parameters } & \multicolumn{1}{c|}{ Values } \\
\hline Area & $2000 \times 2000 \mathrm{~m}^{2}$ & Numbers of Iterations & 10 \\
\hline Numbers of nodes & 40 & Shape of Region & Square \\
\hline Transmission Range & $300 \mathrm{~m}$ & Packet duration & $.01 \mathrm{sec}$ \\
\hline Mobility Model & Random Walk & Packet Size & 512 bytes \\
\hline Speed of ad hoc nodes & $2.5 \mathrm{~m} / \mathrm{s}$ & $\begin{array}{l}\text { Number of packet send per } \\
\text { sec }\end{array}$ & 11 \\
\hline
\end{tabular}

\section{Results}

Section 4.1 shows the algorithm to calculate the above mentioned parameters as discussed below.

\subsection{Algorithm to Evaluate Performance}

The algorithm to calculate the various performance metrics is shown as under. We used 40 nodes $(\mathrm{N}=40)$ and define $\mathrm{k}$ as number of nodes acting as $\mathrm{SU}$. To calculate the value of PoR we use a variable count. To calculate the total no of paths existing between all Sources - Destination (S-D) pairs all combinations of SD pairs are explored. If the path exists between S-D pair the value of count variable is incremented by 1 . For calculating the value of average hop count the P_length variable is used (initialized to zero). If path exists between pair of S-D then the value of hop count is added to $P_{-}$length variable. The process is repeated for all S-D pairs. For calculating PDR the source sends 100 packets using procedure send_data() between every S-D pair and returns successfully packets received by destination. A variable called Cum_Data_packet is used to find cumulative value of packet received by destination. The average hop count, PoR and PDR are calculated by using formula given in Algorithm. 


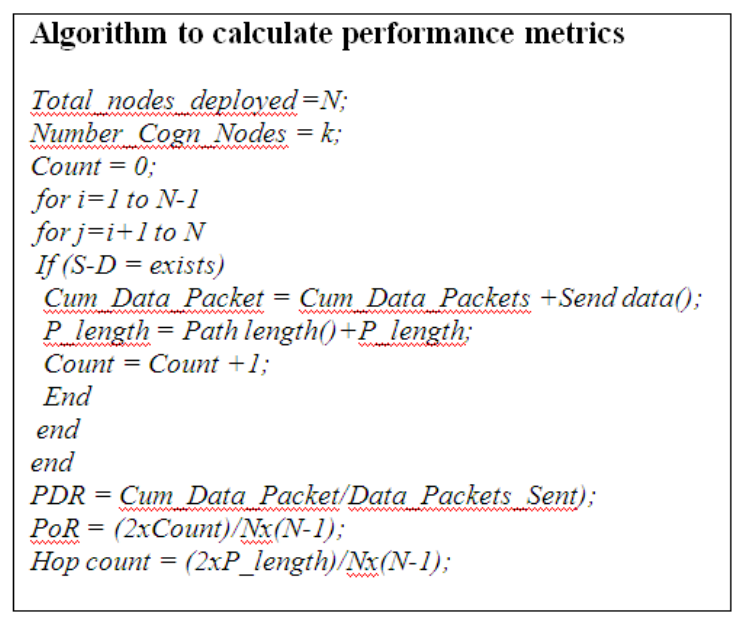

\subsection{Impact on Packet Delivery Ratio (PDR)}

Figure 3 shows the impact of varying number of PU's on PDR. The following inferences can be drawn:

- When the scenario is Ad hoc i.e., the PU doesn't allow SU to use its frequency band, the value of PDR is almost same at various concentration level of PU. This is due to the fact that the path formed is of low hop count value. Moreover the number of paths formed is also very low since PU doesn't allow SU to communicate.

- As the time allocated by the PU to SU increases the value of PDR increases. It reaches to almost $78 \%$ for $50 \%$ concentration level of PU when the time allocated by SU is nearly $50 \%$ of the total time.

- There is some abruption in the results which is due to the fact that the scenario is random.

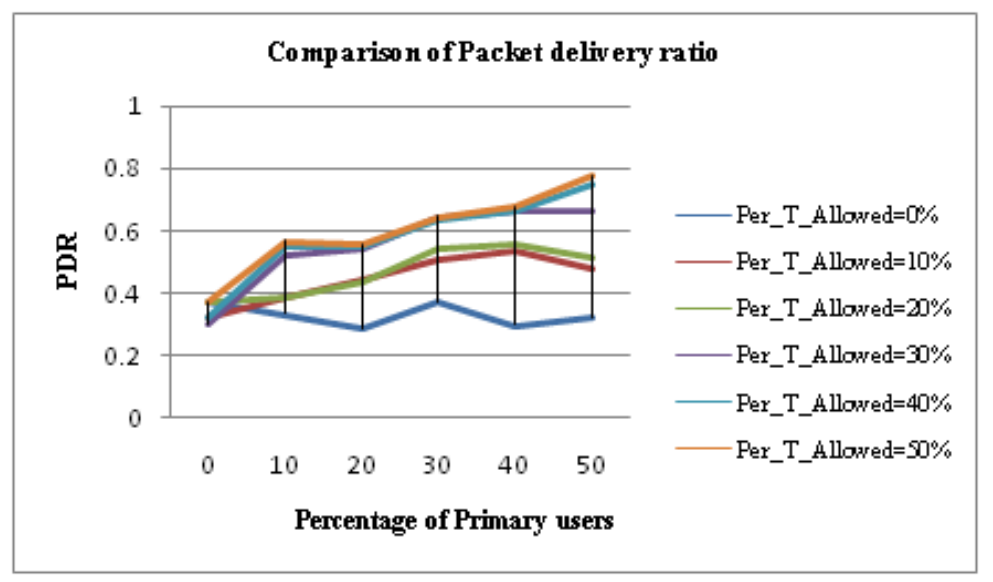

Figure 3. Impact of Primary Node Concentration on PDR

\subsection{Comparison of Hop count}

Figure 4 shows the hop count value for different concentration of PU's and by varying time allowed by PUs to SUs. The following inference can be drawn as follows:

- The value of hop count is lowest for ad hoc network. This is due to the fact that route request packet sent by the SU's are mostly dropped by the PU's.

- With increase in allowable time and concentration of PU's, the value of hop count increases implying that path with higher number of intermediate nodes may exist. 


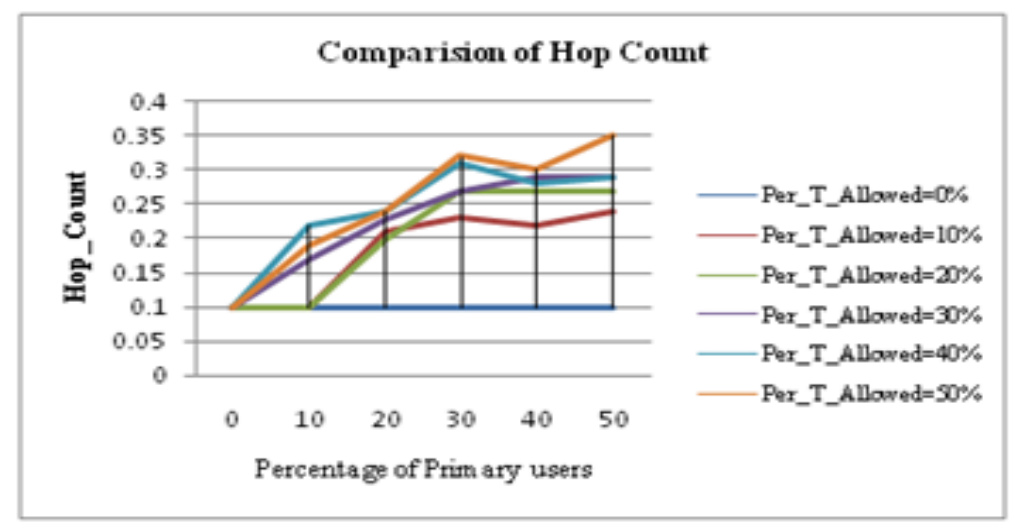

Figure 4. Impact of Primary Nodes Concentration on Hop Count

\subsection{Comparison of Probability of Reach ability (PoR)}

Figure 5 shows the reach ability value by varying percentage of PUs and by varying time allowed by PUs to SUs. An interesting point to be noted here is that there is no significant trend observed with increase in number of PU's. This is due to the reason that the PoR value depends upon the existence of a path.

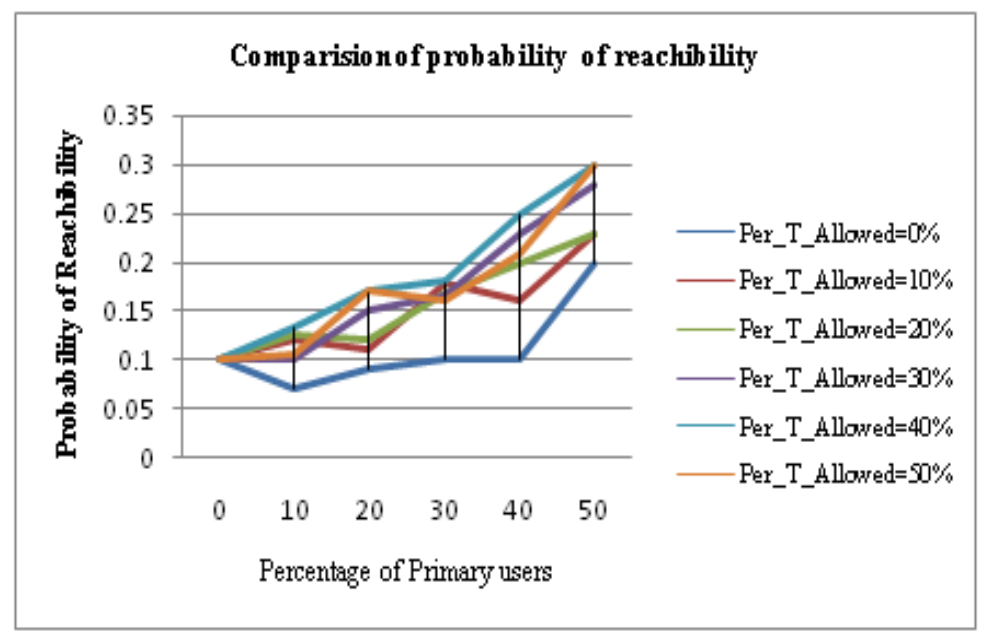

Figure 5. Impact of Primary Nodes Concentration on PoR

\subsection{Comparison of Path Optimality}

Figure 6 shows the path optimality results. The following inference can be drawn

- The path length increases by nearly five time with increase in number of PU's and allowable time.

- The path length shows an increasing trend with increase in number of PU's and allowable time. 


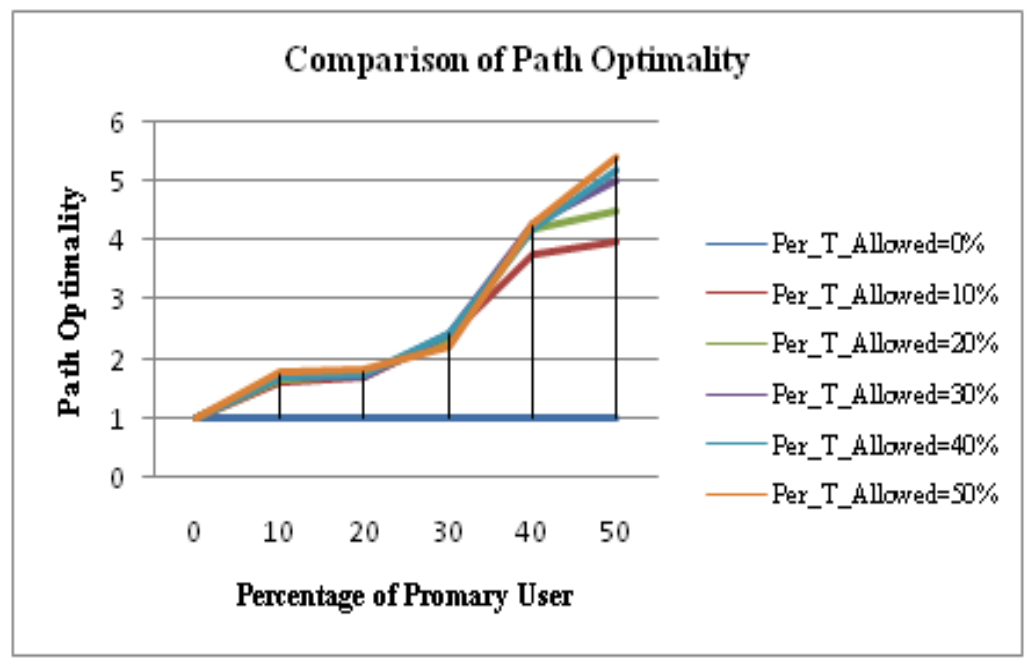

Figure 6. Impact of Primary Nodes Concentration on Path Optimality

\section{Conclusion}

This paper surveys the impact on the routing protocols performance using cognitive radio. A comparison for the use of shortest path routing protocol in Cognitive and pure ad hoc network is presented in this paper. The following points can be inferred from the paper:

- The PDR, PoR hop count and path optimality values in ad hoc network remains almost constant but a sharp increase is seen in the value for Cognitive networks, as number of PU nodes is increased.

- In today's world where there is a scarcity of spectrum availability, the cognitive routing can play a significant role in improving the performance of routing protocols.

The cognitive radios can certainly help in improving performance of routing protocols. The results can be very fruitful for the researchers working in the field of cognitive networks.

\section{References}

[1] S. Gupta, C. K. Nagpal and C. Singla, "Impact of Selfish Node Concentration in Manets", An international journal of wireless and Mobile networks (IJWMN), vol. 3, no. 2, (2011) April.

[2] Y. Chang, L. Kwang, C. Geoffrey, "Cognitive Radio Networking and Communications: An Overview", IEEE Transactions on Vehicular Technology, vol. 60, no.7, (2011) September.

[3] S. M. Kamruzzaman, E. Kim, and D. G. Jeong, "An Energy Efficient Quos Routing Protocol For Cognitive Radio And Ad Hoc Networks", Department of Electronics Engineering, Hanukkah University of FS, Korea, (2011) February 13.

[4] Y.S. Chen and S. H. Liao, "Spectrum Aware Routing in Discontinuous Orthogonal Frequency Division Multiplexing-Based Cr Ad-hoc Networks". Department of computer science and IT, National Taipei University, Taiwan, (2012) January.

[5] A.C. Talay and D.T. Altilar, "United Nodes: Cluster Based Routing Protocol for Mobile Cognitive Radio Networks", Department of Computer engineering, Istanbul Technical University, Turkey, (2011) June.

[6] D. Datla, A. M. Wyglinski, and G. J. Minden, "A Spectrum Surveying Framework For Dynamic Spectrum Access Networks," IEEE Trans. Veh. Technol., vol. 58, no. 8, (2009) October, pp. 41584168.

[7] J. Mitola and G. Q. Maguire, "Cognitive Radio: Making Software radios more Personal", IEEE Pers. Commun., vol. 6, no. 4, (1999) August, pp. 13-18.

[8] J. Mitola, "Cognitive Radio: An Integrated Agent Architecture for software-Defined Radio", Ph.D. dissertation, Roy. Inst. Technology, Stockholm, Sweden, (2000) May.

[9] S. Haykin,"Cognitive Radio: Brain-Empowered Wireless Communications", IEEE J. Sel. Areas Commun. vol. 23, no. 2, (2005) February, pp. 201-220. 
[10] F. Akyildiz, W. Y. Lee, M. Vuran, and S. Mohanty, "Next generation / Dynamic Spectrum Access / Cognitive Radio Wireless Networks: A Survey", Comput. Netw., vol. 50, no. 13, (2006) September, pp. 2127-2159.

[11] A. Sampath, L. Yang, L. Cao, H. Zheng, and B. Y. Zhao, "High Throughput Spectrum-Aware Routing For Cognitive Radio networks", Invited Paper, CrownCom, (2008) May.

[12] T. Fujii and Y. Yamao, "Multi-Band Routing for Ad Hoc Cognitive Radio networks", in Proc. SDR Tech. Conf.,(2006) November.

[13] H. Ma, L. Zheng, X. Ma, and Y. Luo, "Spectrum Aware Routing For Multi-Hop Cognitive Radio Networks With A Single Transceiver", in Proc. CrownCom, (2008) May.

[14] K. C. Chen, B. K. Cetin, Y. C. Peng, N. Prasad, J. Wang, and S. Lee, "Routing For Cognitive Radio Networks Consisting Of Opportunistic links", Wireless Commumn.Mob.Comput., vol. 10, no. 4, (2010) April, pp.451-466.

[15] http://gigaom.com/2010/02/17/analyst-spectrum-shortage-will-strike-in-2013. 